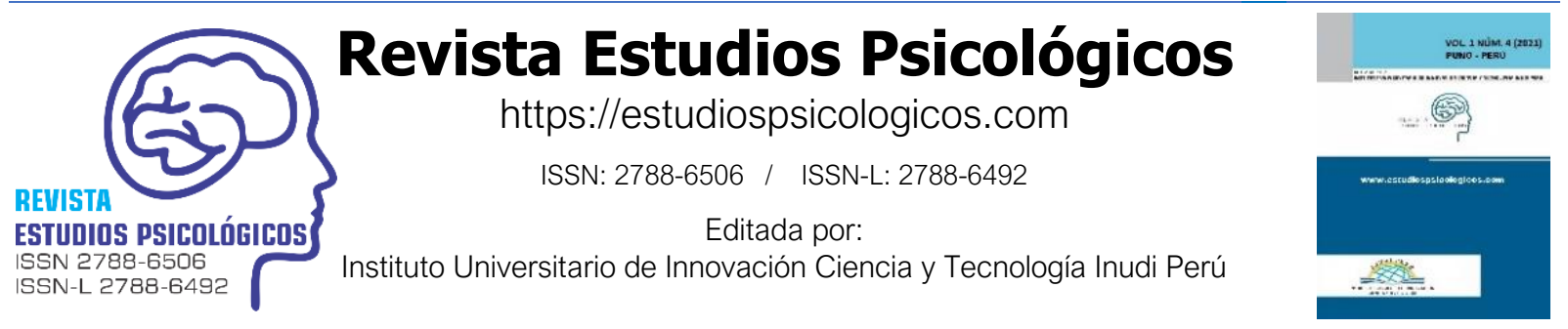

ARTÍCULO ORIGINAL

\title{
Implicaciones del síndrome post COVID-19 en la salud mental en la etapa adulta
}

\author{
Implications of the post COVID-19 syndrome on mental health in the adult stage
}

Implicações da síndrome pós COVID-19 na saúde mental na fase adulta

\author{
Victor Guzmán-Brand ${ }^{1}$ \\ Corporación Unificada Nacional de Educación Superior, Bogotá D.C. - Cundinamarca, Colombia \\ (iD) https://orcid.org/0000-0002-6051-3153
}

D0I: https://doi.org/10.35622/j.rep.2022.02.002

Enviado: 26/11/2021/ Aceptado: 25/02/2022

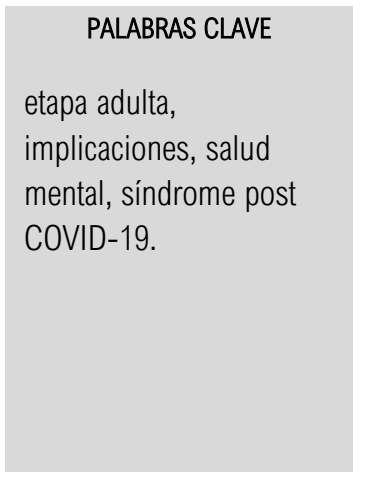

KEYWORDS

adult stage, implications, mental health, postCOVID-19 syndrome.

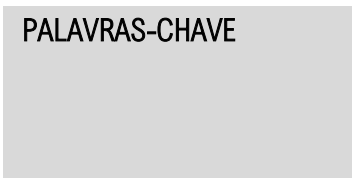

RESUMEN. Los efectos a largo plazo de la enfermedad respiratoria por el virus SARS-CoV-2 en la población adulta es una situación que genera preocupación, porque altera aspectos físicos y mentales del paciente al punto de propiciar una incapacidad en sus funciones cotidianas. Por lo tanto, esta investigación tiene el propósito de comprender las implicaciones del síndrome post COVID-19 en la salud mental en la etapa adulta de la vida. Para la estructuración de la metodología se utiliza la declaración PRISMA 2020 la cual proporciona una síntesis clara de la información del tema en estudio. Entre las conclusiones encontramos que la afectación a largo plazo tiene consecuencias a nivel psicológico y psiquiátrico manifestado en dificultades emocionales en todas las actividades diarias, expresadas en tristezas, bajo nivel de ánimo y percepción de los logros, desmejora la calidad de vida, afecta el estado funcional, la cognición y llega a producir discapacidad importante.

\begin{abstract}
The long-term effects of the respiratory illness caused by the SARS-CoV-2 virus in the adult population is a situation that generates concern because it alters the physical and mental aspects of the patient to the point of providing an inability in their daily functioning. Therefore, this investigation aims to understand the implications of the post-COVID-19 syndrome on mental health in the adult stage of life. For the methodology structuring, the PRISMA 2020 statement is used, which provides a clear summary of the information on the topic in the study. Among the conclusions, we find that the wide-ranging affectation has consequences at a psychological and psychiatric level, manifested in emotional difficulties in all daily activities, expressed in sadness, low level of spirit and perception of achievements, desmejora the quality of life, affects the state functional, cognition and ability to produce critical disability.
\end{abstract}

RESUM0. Os efeitos da ampla praça da doença respiratória pelo vírus SARS-CoV-2 na população adulta é uma situação que gera preocupação, porque altera aspectos físicos e mentais do paciente ao ponto de propiciar uma pessoa incapacitada em suas funções cotidianas. Por isso, esta investigação tem o propósito de compreender as implicações da síndrome pós COVID-19 na saúde

\footnotetext{
${ }^{1}$ Correspondencia: victora.guzman@cun.edu.co
} 
fase adulta, implicações, saúde mental, síndrome pós-COVID-19. mental na etapa adulta da vida. Para a estruturação da metodologia, se utiliza a declaração PRISMA 2020, o qual fornece uma síntese clara das informações do tema em estúdio. Entre as conclusões encontradas que a afetação ao largo prazo tem consequências a nível psicológico e psiquiátrico manifestado em dificuldades emocionais em todas as atividades diárias, expressas, baixo nível de ânimo e percepção dos logros, desmejora a qualidade de vida, afeta o estado funcional, la cognição y lega a produzir deficiência importante.

\section{INTRODUCCIÓN}

La enfermedad infecciosa ocasionada por el virus SARS-CoV-2 conocida como COVID-19 provoca en la mayoría de las personas contagiadas una afección a la salud de manera leve o moderada, pero el riesgo de presentar un nivel grave de la enfermedad es mayor en pacientes con enfermedades de base o subyacentes, sin embargo, este nivel puede producirse en cualquier persona. Según la World Health Organization (2022) se tiene registro hasta le fecha (24 de febrero) de más de 422 millones de casos de contagios confirmados y más de 5,8 millones de muertes relacionadas con la enfermedad, siendo la variante Ómicron y Delta las de mayor prevalencia en todo el mundo, en el continente americano se tienen 146.449 .865 casos de personas contagiadas y 2.618.433 muertes acumuladas, donde según la PanAmerican Health Organization (2021) son Argentina, Bolivia, Brasil, Chile, Colombia y Ecuador son las naciones que han manifestado un importante índice de fallecimientos por COVID-19 en la Región.

Por otro lado, las naciones no estaban preparados económica ni logísticamente para afrontar las implicaciones que acarrearía la pandemia recargando los gastos en el sistema de salud al punto de su debilitación y reorganización. Ahora se presenta la discusión frente a la alta asistencia a los servicios de salud mental y apoyo psicosocial como resultado de forma directa o indirecta de la enfermedad, donde el $52 \%$ de los países en la región manifiestan que tienen fondos parciales o nulos para atender el hecho.

En consecuencia, la pandemia por el COVID-19 a golpeado de manera sustancial la salud mental de las personas produciendo sentimientos de miedo, preocupación y estrés ante las circunstancias adyacentes de la enfermedad. Este hecho es observado con intranquilidad por la PanAmerican Health Organization (2021) por que en la región de las Américas existe un repunte en las personas con casos de angustia, depresión, ansiedad y el insomnio etc., así mismo problemas neurológicos y mentales asociados a la enfermedad después de la recuperación.

Estas implicaciones son conocidas según los estudios como el síndrome post COVID-19, COVID-19 postagudo, COVID prolongado, COVID persistente, COVID de larga duración, etc., que consiste para los Centros para el Control y la Prevención de Enfermedades (2021) como afecciones recurrentes 0 en curso que los pacientes padecen luego de cuatro semanas de la recuperación por el virus. Además, las Organizaciones de Naciones Unidas (2021) manifiestan que las personas pueden llegar a tener consecuencias de forma prolongada en los en los sistemas pulmonar, cardiovascular y nervioso, también repercusiones psicológicas. El objetivo del presente trabajo de revisión sistemática es el de comprender las implicaciones del síndrome post COVID-19 en la salud mental en la etapa adulta de la vida, al ser este segmento de la población la más afectada por esta situación. 


\section{MÉTODO}

La estructura de la metodología está construida en base a una revisión sistemática en razón a las directrices de la declaración PRISMA 2020 permitiendo recolectar y sintetizar las investigaciones, además, de la evaluación del método y fiabilidad de los resultados sobre el tema en estudio. De manera que los documentos para la síntesis cualitativa se toman de fuentes primarias de información siendo artículos de revistas científicas, libros, organizaciones, etc., los cuales fueron publicados en los últimos cinco años, en las áreas de la salud, están disponibles de forma abierta. Se utilizó para la exploración bases de datos por ejemplo PubMed, ScienceDirect, Dialnet de igual modo motores de búsqueda como Google Académico y RefSeek.

Conjuntamente, para impedir una sobrecarga y hacer selectiva la búsqueda de información de manera avanzada se utilizó filtros de elección acompañados con sus operadores lógicos como: síndrome post COVID-19, COVID19 postagudo, COVID prolongado, COVID de larga duración. Para decir los documentos que cumplen con los criterios de inclusión, investigaciones que evaluaran pacientes en contextos clínicos, publicaciones en español o inglés, descartando investigaciones en menores de edad, que no tuvieran en cuenta las implicaciones en salud mental, , no relacionadas con el tema, artículos de opinión o difusión, por último, se realizó una selección de manera independiente se excluyeron trabajos con títulos no relacionados, duplicados y luego de leer el resumen no sean relevantes para la investigación, estrategia expuesta en la siguiente tabla:

\section{Tabla 1}

\section{Ecuaciones de búsqueda y bases de datos empleadas en la investigación.}

\begin{tabular}{|c|c|c|c|c|c|c|c|c|}
\hline $\begin{array}{l}\text { BASE DE DATOS/ } \\
\text { ECUACIONES DE } \\
\text { BÚSQUEDA }\end{array}$ & $\begin{array}{l}\text { Síndrome } \\
\text { post } \\
\text { COVID-19 }\end{array}$ & $\begin{array}{l}\text { COVID-19 } \\
\text { postagudo }\end{array}$ & $\begin{array}{l}\text { COVID } \\
\text { prolongado }\end{array}$ & $\begin{array}{l}\text { COVID de } \\
\text { larga } \\
\text { duración }\end{array}$ & $\begin{array}{l}\text { Exclusión } \\
\text { por título no } \\
\text { relacionado }\end{array}$ & $\begin{array}{l}\text { Exclusión } \\
\text { después } \\
\text { de leer el } \\
\text { resumen }\end{array}$ & $\begin{array}{l}\text { Exclusión } \\
\text { por estar } \\
\text { duplicadas }\end{array}$ & $\begin{array}{l}\text { Total, } \\
\text { escogidas } \\
\text { para el } \\
\text { estudio }\end{array}$ \\
\hline PubMed & 20 & 3 & 121 & 0 & 99 & 30 & 6 & 9 \\
\hline ScienceDirect & 66 & 6 & 290 & 134 & 439 & 45 & 1 & 6 \\
\hline Dialnet & 26 & 1 & 74 & 38 & 117 & 20 & 0 & 2 \\
\hline RefSeek & & & & & & & & 2 \\
\hline Libros & & & & & & & & 3 \\
\hline Páginas Web & & & & & & & & 3 \\
\hline Organización & & & & & & & & 2 \\
\hline Total & 112 & 10 & 485 & 172 & 655 & 95 & 7 & $779-27$ \\
\hline
\end{tabular}

Nota: En la tabla se observa los elementos obtenidos de las bases de datos y otras fuentes utilizadas para la síntesis cualitativa. 


\section{Figura 1}

Proceso de selección según el diagrama de flujo PRISMA 2020

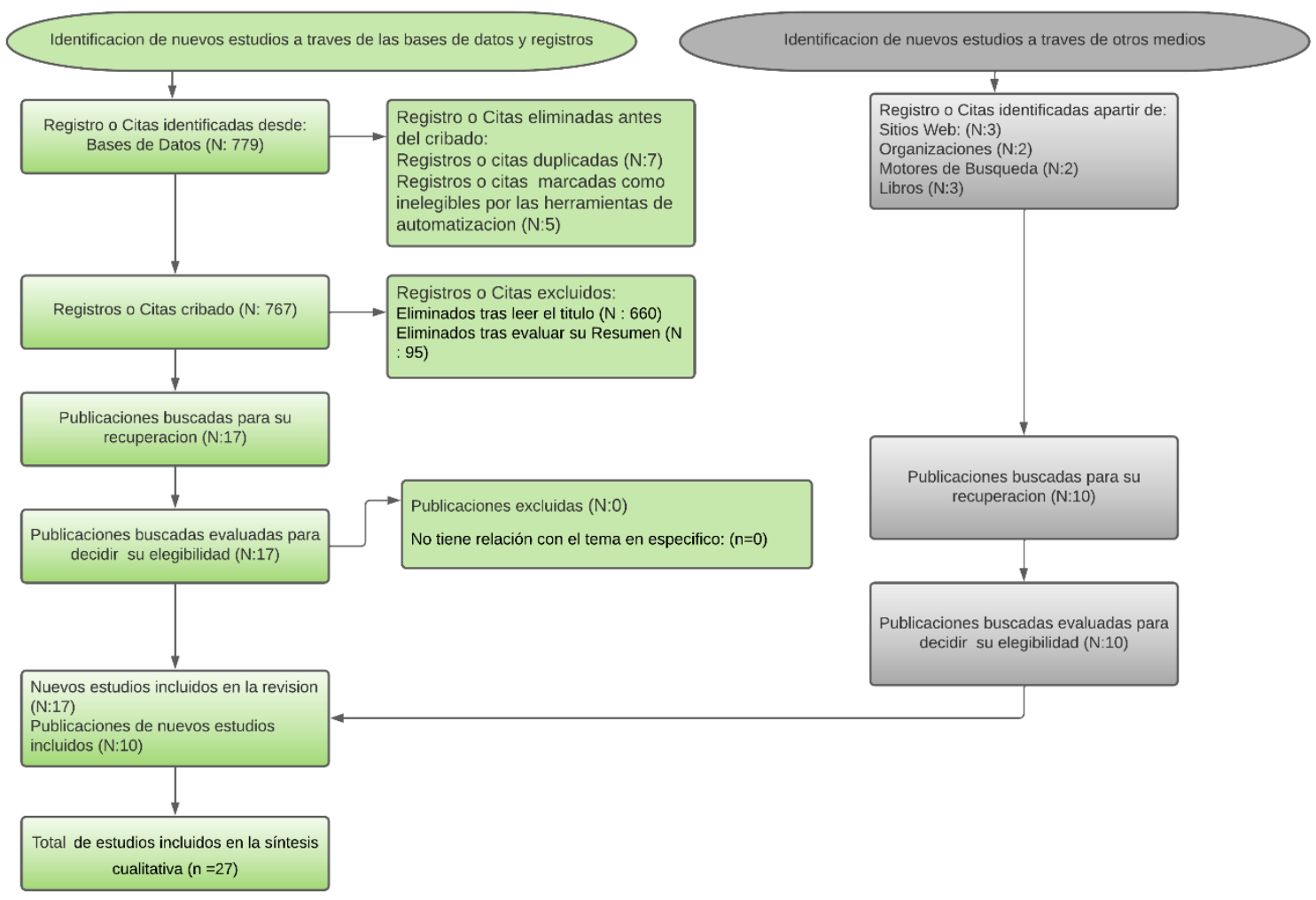

Nota: Esquema tomado Page et al., 2021, articulo declaración PRISMA 2020: una guía actualizada para la publicación de revisiones sistemáticas, Diagrama de flujo PRISMA 2020, D0I: 10.1016/j.recesp.2021.06.016.

Tabla 2

Estudios sobre el síndrome post-COVID-19 incluidos en la síntesis cualitativa.

\begin{tabular}{|c|c|c|c|c|c|c|c|}
\hline Año & Autor & Gravedad & $\begin{array}{l}\text { Tiempo/d } \\
\text { espues }\end{array}$ & $\begin{array}{l}\text { Clase } \\
\text { estudio }\end{array}$ & $\begin{array}{l}\text { Prevalenc } \\
\text { ia }\end{array}$ & Instrumento & Síntomas comunes \\
\hline 2022 & Ayuso et al. & $\begin{array}{l}\text { Moderado } \\
\text { Grave }\end{array}$ & $\begin{array}{l}40 \\
\text { semanas }\end{array}$ & $\begin{array}{l}\text { Transversa } \\
\text { I } \\
443 \\
\text { participant } \\
\text { es }\end{array}$ & $36,8 \%$ & $\begin{array}{ll}\text { - } & \text { Encuesta telefónica } \\
\text { - } & \text { Escala EQ-5D } \\
\text { - } & \text { La escala EQ-EVA } \\
\text { global } \\
\text { Y EQ-Health Index global. }\end{array}$ & $\begin{array}{l}\text { Ansiedad/depresión (23,9\%) } \\
\text { y la movilidad }(16,5 \%) .\end{array}$ \\
\hline 2022 & González & $\begin{array}{l}\text { Leve } \\
\text { Moderada }\end{array}$ & $\begin{array}{l}4 \text { a } 12 \\
\text { semanas }\end{array}$ & $\begin{array}{l}\text { Trasversal } \\
1.366 \\
\text { participant } \\
\text { es }\end{array}$ & $64,3 \%$ & $\begin{array}{l}\text { - Encuesta } \\
\text { - Escala de Fatiga de } \\
\text { Chalder (CFQ-11) }\end{array}$ & $\begin{array}{l}\text { Cansancio en el } 67,3 \% \\
\text { cefalea } 45,2 \% \text {, el dolor } \\
\text { corporal } 42,3 \% \text { trastornos } \\
\text { del sueño } 36,5 \% \text {. Ansiedad } \\
(26,06 \%) \text { de y síntomas } \\
\text { depresivos en el }(18,08 \%)\end{array}$ \\
\hline 2022 & Valverde et al. & Grave & $\begin{array}{l}12 \\
\text { semanas }\end{array}$ & $\begin{array}{l}\text { Descriptiv } \\
0 \\
59 \\
\text { pacientes }\end{array}$ & $84 \%$ & $\begin{array}{ll}\text { - } & \text { Entrevista } \\
\text { - } & \text { El Cuestionario } \\
& \text { Salud SF-36 }\end{array}$ & $\begin{array}{l}59 \% \text { tenían disnea, y el } 78 \% \\
\text { tenían otros síntomas } \\
\text { persistentes, cansancio y } \\
\text { dolores musculares, } \\
\text { ansiedad y/o depresión. }\end{array}$ \\
\hline 2021 & $\begin{array}{l}\text { Rodríguez et } \\
\text { al. }\end{array}$ & Grave & $\begin{array}{l}4 \quad-6 \\
\text { semanas }\end{array}$ & $\begin{array}{l}\text { Descriptiv } \\
0\end{array}$ & $87,93 \%$ & - $\quad$ Test Euroqol-5D-3L. & $\begin{array}{l}28 \% \text { ansiedad y depresión. } \\
20 \% \text { presenta TEPT. }\end{array}$ \\
\hline
\end{tabular}




\begin{tabular}{|c|c|c|c|c|c|c|c|}
\hline & & & & $\begin{array}{l}29 \\
\text { pacientes }\end{array}$ & & $\begin{array}{l}\text { - } \text { Test de } 6 \text { minutos de } \\
\text { - } \quad \text { Deterioro cognitivo } \\
\text { (MoCA). } \\
\text { - } \text { Escala de ansiedad y } \\
\text { depresión hospitalaria } \\
\text { (HADS). } \\
\text { Escala de trauma de } \\
\text { Davidson. } \\
\text { Escala analógica visual } \\
\text { (EVA). }\end{array}$ & $\begin{array}{ll}\text { Alteración del } & \text { estado } \\
\text { cognitivo } & \\
\text { en el 62\%. } & \\
21 \% \text { presento } & \text { dolor } \\
\text { neuropático. } & \end{array}$ \\
\hline 2021 & Anaya et al. & $\begin{array}{l}\text { Moderada } \\
\text { Grave }\end{array}$ & 4 semanas & $\begin{array}{l}\text { Descriptiv } \\
0 \\
116 \\
\text { pacientes }\end{array}$ & $20 \%$ & $\begin{array}{ll}\text { - } & \text { Encuesta } \\
\text { - } & \text { Escalas Zung }\end{array}$ & fatiga posviral con el $70 \%$ \\
\hline 2021 & $\begin{array}{l}\text { Mahmud et } \\
\text { al. }\end{array}$ & $\begin{array}{l}\text { Leve } \\
\text { Moderada } \\
\text { Grave }\end{array}$ & 4 semanas & $\begin{array}{l}\text { Prospectiv } \\
0 \\
400 \\
\text { pacientes }\end{array}$ & $46 \%$ & - Entrevista & $\begin{array}{l}\text { La fatiga posviral (33 } \\
\%) \text {. Otras características } \\
\text { incluyeron tos persistente } \\
(8,5 \%) \text {, disnea postesfuerzo } \\
(7 \%) \text {, dolor de cabeza }(3,4 \\
\%) \text { vértigo }(2,3 \%) \text { y } \\
\text { trastornos relacionados con } \\
\text { el sueño }(5,9 \%)\end{array}$ \\
\hline 2021 & $\begin{array}{l}\text { Barbagelata et } \\
\text { al. }\end{array}$ & Moderada & $\begin{array}{l}45 \text { a } 120 \\
\text { días }\end{array}$ & $\begin{array}{l}\text { Trasversal } \\
200 \\
\text { pacientes }\end{array}$ & $56 \%$ & $\begin{array}{l}\text { - Escala de estado } \\
\text { funcional post-COVID- } \\
19\end{array}$ & $\begin{array}{l}\text { Menos probabilidades de } \\
\text { alcanzar el umbral } \\
\text { anaeróbico }(50,9 \%)\end{array}$ \\
\hline 2021 & $\begin{array}{l}\text { Rodríguez et } \\
\text { al. }\end{array}$ & $\begin{array}{l}\text { Leve } \\
\text { Moderada }\end{array}$ & $\begin{array}{ll}4 & -6 \\
\text { semanas }\end{array}$ & $\begin{array}{l}\text { Trasversal } \\
1834 \\
\text { participant } \\
\text { es }\end{array}$ & $95,9 \%$ & - $\quad$ Encuesta & $\begin{array}{l}95,9 \% \text { astenia, } 86,5 \% \\
\text { cefalea, 86,2 \% bajo estado } \\
\text { de ánimo, 82,8 \% mialgias, } \\
79,3 \% \text { disnea,70,8 \% } \\
\text { diarrea y } 69,9 \% \\
\text { palpitaciones }\end{array}$ \\
\hline 2021 & Davis et al. & $\begin{array}{l}\text { Leve } \\
\text { Moderada }\end{array}$ & $\begin{array}{l}4 \quad-6 \\
\text { semanas }\end{array}$ & $\begin{array}{l}\text { Trasversal } \\
3762 \\
\text { participant } \\
\text { es }\end{array}$ & $85,9 \%$ & - Encuesta & $\begin{array}{l}86,7 \% \text { fatiga, } 88 \% \\
\text { problemas de memoria. }\end{array}$ \\
\hline 2020 & Goërtz et al. & $\begin{array}{l}\text { Leve } \\
\text { Moderada }\end{array}$ & 79 días & $\begin{array}{l}\text { Descriptiv } \\
0 \quad 669 \\
\text { participant } \\
\text { es }\end{array}$ & $93 \%$ & - Encuesta & $\begin{array}{l}\text { Fatiga } 87 \% \text {; disnea } 71 \% \text {; } \\
\text { dolor torácico } \\
44 \% \text {; cefalea } 38 \% \text {; dolor } \\
\text { muscular } \\
36 \% \text {; palpitaciones } 32 \% \text {, y } \\
\text { tos } 29 \%\end{array}$ \\
\hline 2020 & Agustin et al. & $\begin{array}{l}\text { Leve } \\
\text { Moderada }\end{array}$ & $\begin{array}{l}4 \text { y } 7 \\
\text { meses }\end{array}$ & $\begin{array}{l}\text { Prospectiv } \\
0 \\
958 \\
\text { participant } \\
\text { es }\end{array}$ & $27,8 \%$ & - Encuesta & $\begin{array}{l}8,6 \% \text { dificultad respiratoria, } \\
12,4 \% \text { anosmia, } 11,1 \% \\
\text { ageusia, 9,7\% fatiga }\end{array}$ \\
\hline 2022 & $\begin{array}{l}\text { Staub y } \\
\text { Portella }\end{array}$ & & & & & Revisión Literatura & \\
\hline 2021 & Carod & & & & & Revisión Literatura & \\
\hline 2021 & Guerrero & & & & & Revisión Literatura & \\
\hline 2021 & Castillo et al. & & & & & Revisión Literatura & \\
\hline
\end{tabular}




\begin{tabular}{lll}
\hline 2021 & Cimas & Revisión Literatura \\
\hline 2021 & López et al. & Revisión Literatura \\
\hline
\end{tabular}

Nota: En la tabla se describe los artículos científicos, libros y otras fuentes tomadas para la construcción de la síntesis.

\section{RESULTADOS}

\section{Conceptos Generales}

EI SARS-CoV-2 es el virus causante del síndrome respiratorio agudo severo que infecta de manera sistémica y afecta diferentes órganos a la vez. Por lo tanto, para Guerrero y Bilbao (2021) el síndrome post COVID-19 puede producirse en razón a que:

El virus puede permanecer latente en algún reservorio (tejido o célula del sistema inmunitario) y reactivarse periódicamente al producirse cambios en la homeostasis del sistema inmunitario, lo que causa brotes de sintomatología, pero no hace que el virus sea detectable en las vías altas (p.90).

Igualmente, Pardo y López (2021) proponen que las causas del COVID prolongado obedecen a " respuestas inflamatorias graves, microangiopatía trombótica, tromboembolia venosa y falta de oxígeno" (p.7). Por otro lado, Augustin et al. (2021) enfatiza que este síndrome se observa en mayor medida en personas que han presentado nivel modero o grave de la enfermedad.

El síndrome post COVID-19 es descrito como una prolongación o una extensión en el tiempo de los signos y síntomas clínicos generados por el contagio del virus SARS-CoV-2 en las personas recuperadas del nivel grave 0 moderado de la enfermedad, además, observables después de 12 semanas de estar contagiados. Para la National Institute for Health and Care Excellence - NICE (2020) define el síndrome y sus síntomas como:

1. COVID-19 agudo: Signos y síntomas de COVID-19 hasta por 4 semanas.

2. COVID-19 sintomático en curso: Signos y síntomas de COVID-19 desde las 4 semanas hasta las 12 semanas.

3. Síndrome post-COVID-19: Los signos y síntomas que se desarrollan durante o después de una infección compatible con COVID-19, continúan durante más de 12 semanas y son no explicado por un diagnóstico alternativo.

\section{Tabla 3}

\section{Síntomas del síndrome post COVID-19}

\begin{tabular}{|c|c|}
\hline Sistema & Síntomas \\
\hline Respiratorio & $\begin{array}{ll}\text { - } & \text { Falta de aliento } \\
\text { - } & \text { Tos }\end{array}$ \\
\hline Cardiovascular & $\begin{array}{l}\text { - } \text { Opresión en el pecho } \\
\text { - } \quad \text { Dolor en el pecho } \\
\text { - } \quad \text { Palpitaciones }\end{array}$ \\
\hline Generalizado & $\begin{array}{l}\text { - } \quad \text { Fatiga } \\
\text { - } \quad \text { Fiebre } \\
\text { - } \\
\text { Dolor }\end{array}$ \\
\hline Neurologico & $\begin{array}{l}\text { - Deterioro cognitivo (niebla mental, perdida de concentración } 0 \\
\text { problemas de memoria) }\end{array}$ \\
\hline
\end{tabular}




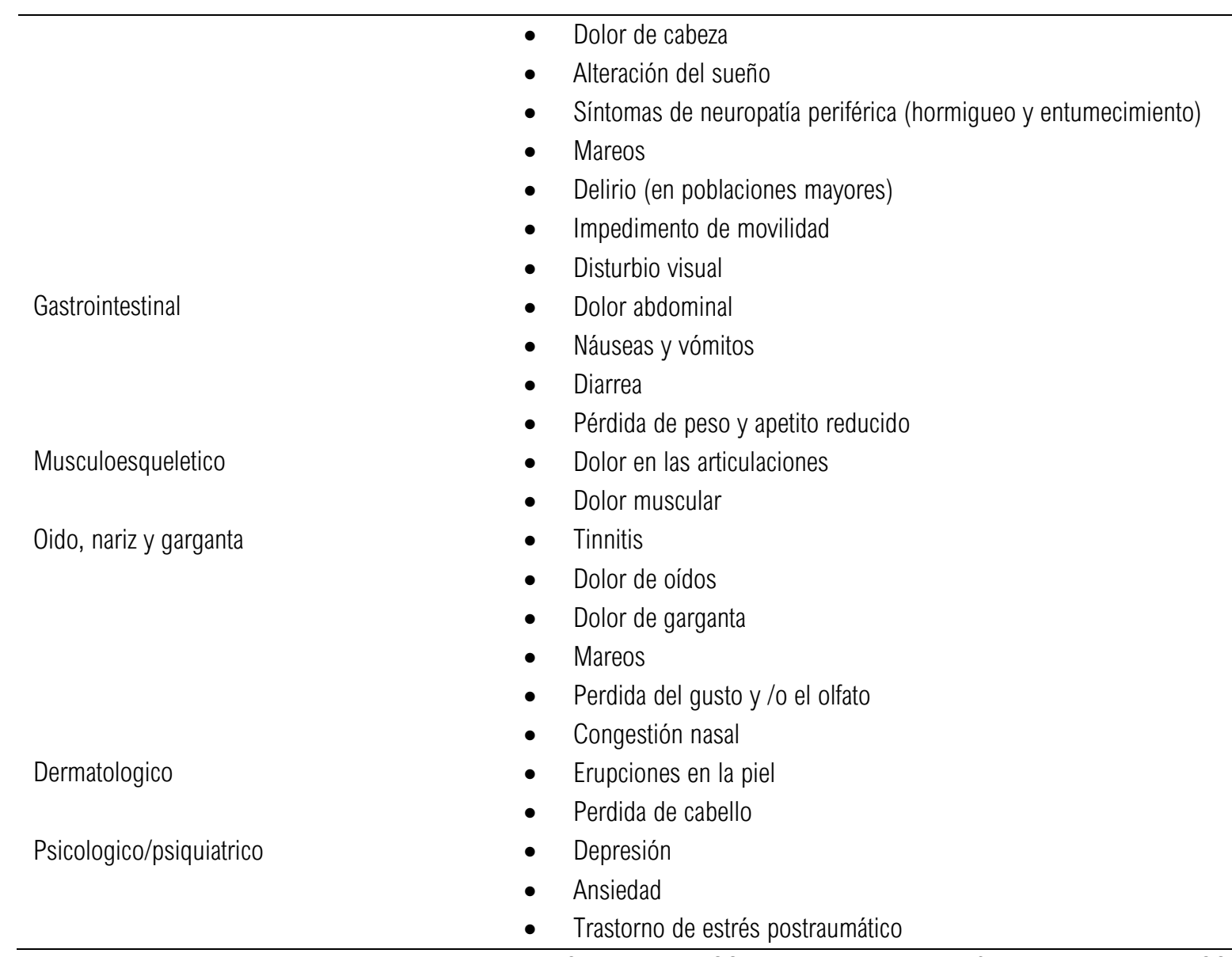

Nota: En la tabla se presenta el esquema de la Guía rápida de COVID-19: manejo de los efectos a largo plazo de COVID-19, National Institute for Health and Care Excellence (NICE), 2021, https://www.nice.org.uk/guidance/ng188

Entre las publicaciones se resaltan estudios como el de Rodríguez et al. (2021) los cuales encuestaron a 1834 pacientes que manifestaron presencia prolongada de signos y síntomas los cuales se agruparon en un 95,9\% generales, 86,5 \% neurológicos,86,2 \% psicológicos/emocionales, 82,8 \% del aparato locomotor, 79,3\% respiratorios y $70,8 \%$ digestivos, siendo los más usuales $95,9 \%$ astenia, $86,5 \%$ cefalea, $86,2 \%$ bajo estado de ánimo, 82,8 \% mialgias, 79,3\% disnea,70,8 \% diarrea y 69,9\% palpitaciones.

De igual manera en la investigación de González (2022) donde cuenta con 1366 participantes el 64, 3\% presentaron síntomas prolongados después de 4 a 6 semanas de haberse contagiado con el virus SARS-CoV2. Donde las afecciones más recurrentes fueron cefalea con 45,2\%, el dolor corporal en el 42,3\% y los trastornos del sueño con 36,5\%, además, ansiedad (26,06\%) y síntomas depresivos en el $(18,08 \%)$. Además, el factor de riesgo más grave es el sedentarismo (40,3\%) seguido de ser trabajador de la salud (11,87\%).

Por otro lado, Davis et al. (2021) en su investigación con la participación de 3762 personas recuperadas del COVID-19 un 85,9\% manifestaron haber experimentado dificultades después de actividad física 0 mental y estrés, adicionalmente $(45,2 \%)$ expresaron que se vio afectado su trabajo ya que requerían un menor tiempo en las actividades. Asimismo, Goërtz et al. (2020) en su estudio con 2113 personas recuperados del virus presenta que los síntomas usuales de fatiga 95\%, disnea 90\%, además los efectos están presentes después de 3 meses. 


\section{Sintomatología Psicológicas y Psiquiátricas Comunes del Síndrome Post Covid-19}

Entre las manifestaciones psicológicas y psiquiátricas comunes del síndrome post COVID-19 por su prevalencia se resaltan los padecimientos por cefaleas, deterioro cognitivo, perturbación del gusto y olfato, trastornos de depresión, ansiedad y del sueño (Castillo et al., 2022). Estos efectos prolongados del virus son estudiados y descritos por Rodríguez et al. (2021) donde realiza un diagnóstico funcional en 29 pacientes que ingresa a UCI de los cuales 9 de 10 supervivientes experimentan alguna alteración por el síndrome post cuidados intensivos después de cuatro a seis semanas de salir del hospital, además, un $20 \%$ se le detectó trastorno de estrés postraumático patológico.

Pese a su recuperación de acuerdo con Valverde et al. (2022) el 15,25\% de los pacientes de su estudio requirieron un nuevo ingreso hospitalario, el 78\% tenían síntomas prolongados como cansancio, dolores musculares, ansiedad y depresión, un 59\% disnea, adicionalmente, solo el 30,5\% efectuaron una reincorporación a las actividades cotidianas, el resto presentaron mayores problemas tanto en las tareas diarias como en el trabajo relacionados con la salud física, asociado a un menor rendimiento. Por otro lado, exhibieron dificultades emocionales en todas las actividades expresadas en tristezas, depresión y ansiedad disminuyendo el tiempo dedicado al trabajo.

Conjuntamente, las indagaciones coinciden que el sexo femenino tiene mayor prevalencia de la enfermedad con un 63,6\% (Augustin et al., 2021; Barbagelata et al., 2021). Por otro lado, Staub y Staub (2022) en su estudio manifiestan que el síndrome inflamatorio multisistémico (SIMS) está asociado al síndrome post COVID-19 detectado especialmente en menores de edad.

Es así pues, como Anaya et al. (2021) plantea en sus estudios que el síndrome post COVID-19 afecta la calidad de vida de los pacientes y tiene unas implicaciones a largo plazo como son fatiga o debilidad muscular, disnea, dolor y malestar, ansiedad, depresión, y dificultades en la concentración problemas que dieron a conocer el $20 \%$ de las personas en tratamiento. En la investigación de Mahmud et al. (2021) se observa cómo el $46 \%$ de Ios participantes presentaron alguna incidencia en razón al COVID postagudo especialmente la fatiga posviral con el 33\%, adicional tos $8,5 \%$, disnea $7 \%$, dolor de cabeza 3,4\%, vértigo 2,3\% y trastorno del sueño 2,3\%.

En consecuencia, la sintomatología psicológicas y psiquiátricas comunes del síndrome post covid-19 llegan a presentarse de manera tardía y perseverar posteriormente al contagio, donde Bouza et al. (2021) expone un estudio realizado en Estados Unidos en donde más de 60.000 personas eran pacientes COVID-19, observando que "más del doble de riesgo de una enfermedad psiquiátrica en los 14 a 90 días posteriores a la infección por SARS-CoV-2, comparado con lo que ocurría con otras enfermedades" (p.5). Por lo tanto, para Augustin et al. (2021) los síntomas consiguen desmejorar la calidad de vida, impactar el estado funcional, la cognición y el ánimo de los pacientes recuperados y provocar una discapacidad importante. 


\section{Figura 2}

\section{Manifestaciones psicosociales de la infección por SARS-CoV-2.}

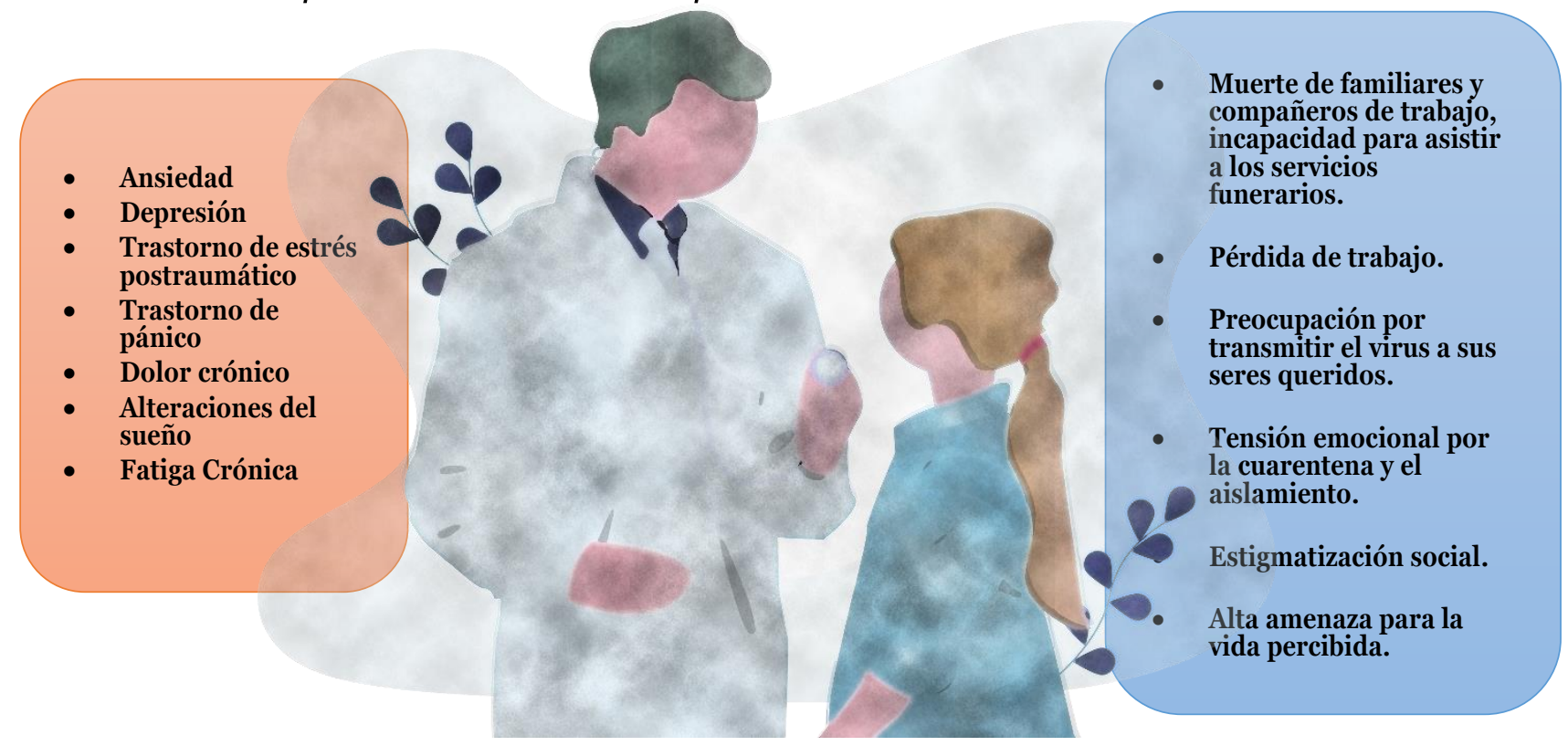

Nota: Tomado del estudio de Higgins et al. (2021) COVID-19: ¿de una enfermedad aguda a una crónica? Posibles consecuencias para la salud a largo plazo. Creación Propia.

\section{Trastornos de Ansiedad y Depresión}

La ansiedad es caracterizada como un comportamiento de alerta exorbitado por un sentimiento de amenaza no motivado, por lo tanto, ocasiona una alteración al sistema nervioso, implica estados de miedo, desesperación 0 de intimidación injustificadas, para Clark y Beck (2012) este estado de temor, angustia e inquietud no son únicos de las catástrofes, calamidades y situaciones que ponen en riesgo la vida, en la mayoría de los hechos se impulsan en ambientes de presiones, requerimientos y estresantes que aparecen en la cotidianidad, según Ander (2016) hay más de un tipo entre las que se encuentran:

- Estado de Ansiedad: Una situación corta que puede pobre llevar cualquier persona.

- Ansiedad de Riesgo: Se presenta una tensión entre los actual y lo posible en el futuro, por lo tanto, es una incapacidad de convivir en el presente.

- Ansiedad Aguda: Es la forma patología que se suele expresar en una falta de sosiego que produce un aumento de la tensión muscular (rigidez, sentirse agobiado) y otras manifestaciones neurovegetativas (sudor, boca seca, sensación subjetiva de peligro, nerviosismo) y en algunas ocasiones, dificultades de concentrarse. (p.26)

Algunos estudios demuestran como estos tipos de trastornos se acentúa en esta clase se síndromes post infecciosos donde M. Rodríguez et al. (2022) expone que las personas que necesitaron asistencia respiratoria el 28\% presenta ansiedad y depresión, por otro lado, Ayuso et al. (2022) manifiesta que el área más afectada en pacientes recuperados por el virus en el nivel moderado son la ansiedad, depresión con el $(23,9 \%$ ) y la movilidad (16,5\%). Además, según López et al. (2021) entre los síntomas psicológicos de mayor persistencia están de un 30-40\% ansiedad y depresión, después el trastorno de estrés postraumático, insomnio y 
sintomatología obsesiva-compulsiva. Para Mahmud et al. (2021) la pandemia del COVI-19 provoco efectos y angustias prolongadas en la mitad de los pacientes.

La depresión se especifica por una tristeza prolongada, acompañada de un desinterés por realizar las tareas cotidianas y placenteras. Esta condición llega a modificar el sueño, cambios en el apetito, produce inactividad y desconcentración. Según la Organización Mundial de la Salud (2022) la depresión es uno de los trastornos más incapacitantes en el mundo. De tal modo que el síndrome post COVID-19 se vincula con episodios de estados de ánimo alterado, tristeza, ansiedad, depresión y cuadros de estrés postraumático primordialmente en trabajadores de la salud y ocupaciones esenciales (Cimas, 2021). En el estudio de P. Rodríguez et al. (2021) muestra como el $20 \%$ de los pacientes que ingresaron a la UCI presentaron trastorno de estrés post traumático, adicional alteración del estado cognitivo en el $62 \%$ y $21 \%$ presento dolor neuropático.

\section{CONCLUSIONES}

El contagio del virus SARS-CoV-2 y sus posteriores niveles de gravedad pueden llegar a prolongar los síntomas, esta situación se presenta en un gran porcentaje en adultos, pero sus efectos también aparecen en menores. Los estudios sobre la incidencia del síndrome post COVID-19 en la salud en la etapa adulta exponen que los factores de riesgo son el sexo femenino, manifestar dificultad respiratoria, tener un prolongado periodo de recuperación y nivel grave de la enfermedad, a su vez el sedentarismo y ser trabajador sanitario. Se demuestra como el síndrome post COVID-19 tiene consecuencias en las capacidades físicas y mentales perturbando el desempeño cotidiano y laboral.

En consecuencia, esta afectación a largo plazo tiene implicaciones a nivel psicológico y psiquiátrico manifestado en dificultades emocionales en todas las actividades diarias, expresadas en tristezas, bajo nivel de ánimo y percepción de sus logros, desmejora la calidad de vida, afecta el estado funcional, la cognición y llega a producir discapacidad importante a la persona. Los trastornos psicológicos comunes son la ansiedad, depresión y estrés post traumático.

Es así como es necesario un seguimiento a los pacientes afectados, mantener una comunicación abierta y brindar un apoyo emocional, orientación de autocuidado y rehabilitación en relación con la actividad física y mental diaria, respiración, dieta e hidratación. Por otro lado, coordinar una intervención multidisciplinar para su atención, además incluir en las campañas de vacunación que previenen los síntomas graves del virus adicionalmente ayuda a contrarrestar las implicaciones en la salud del síndrome post COVID-19. Entre las limitaciones de la revisión sistemática esta la constante evolución de la información científica en cuanto a los efectos e implicaciones del síndrome post COVID-19 por lo actual del tema.

\section{Conflicto de intereses / Competing interests:}

El autor declara que no incurre en conflictos de intereses.

\section{Rol de los autores / Authors Roles:}

No aplica

\section{Fuentes de financiamiento / Funding:}

El autor declara que las fuentes de financiación que dieron lugar a la investigación son de carácter personal y motivación profesional..

\section{Aspectos éticos / legales; Ethics / legals:}


El autor declara no haber incurrido en aspectos antiéticos, ni haber omitido aspectos legales en la realización de la investigación.

\section{REFERENCIAS}

Anaya, J., Rojas, M., Salinas, M., Rodríguez, Y., Roa, G., Lozano, M., Rodríguez-Jiménez, M., Montoya, N., Zapata, E., Monsalve, D., Acosta, Y., \& Ramírez, C. (2021). Post-COVID syndrome. A case series and $\begin{array}{llll}\text { comprehensive review. Autoimmunity Reviews, } & 202947 .\end{array}$ https://doi.org/10.1016/j.autrev.2021.102947

Ander, E. (2016). Diccionario de psicología (tercera). http://hdl.handle.net/20.500.11818/1152

Augustin, M., Schommers, P., Stecher, M., Dewald, F., Gieselmann, L., Gruell, H., Horn, C., Vanshylla, K., Cristanziano, V., Osebold, L., Roventa, M., Riaz, T., Tschernoster, N., Altmueller, J., Rose, L., Salomon, S., Priesner, V., Luers, J., Albus, C., ... Lehmann, C. (2021). Post-COVID syndrome in non-hospitalised patients with COVID-19: a longitudinal prospective cohort study. The Lancet Regional Health - Europe, 6, 35-45. https://doi.org/10.1016/j.lanepe.2021.100122

Ayuso, B., Pérez, A., Besteiro, Y., Romay, E., García, M., Marchán, Á., Rodríguez, A., Corredoira, J., \& Rabuñal, R. (2022). Calidad de vida relacionada con la salud en pacientes recuperados de COVID-19. Journal of Healthcare Quality Research, 7, 67-87. https://doi.org/10.1016/j.jhqr.2022.01.001

Barbagelata, L., Masson, W., Iglesias, D., Lillo, E., Migone, J., Orazi, M., \& Maritano, J. (2021). Cardiopulmonary Exercise Testing in Patients with Post-COVID-19 Syndrome. Medicina Clínica, 5, 45-56. https://doi.org/10.1016/j.medcli.2021.07.007

Bouza, E., Cantón, R., De Lucas, P., García, A., García, A., Gómez, J., González, J., Hernández, T., Martín, M., Martín, F., Martínez, M., Molero, J., Moreno, S., Rodríguez, F., Ruiz, J., De Pablo, S., Porta, J., \& Santos, M. (2021). Post-COVID syndrome: A reflection and opinion paper. Revista Española de Quimioterapia, 34(4), 269-279. https://doi.org/10.37201/req/023.2021

Castillo, F., Fernández, E., Campos, M., \& García, B. (2022). Sintomatología neuropsiquiátrica en el síndrome post-COVID. Propuesta de manejo y derivación desde atención primaria. Medicina de Familia. SEMERGEN, 8, 12-24. https://doi.org/10.1016/j.semerg.2021.09.012

Cimas, J. (2021). Seguimiento de los pacientes con secuelas no respiratorias de la COVID-19. FMC - Formación Médica Continuada en Atención Primaria, 28(2), 81-89. https://doi.org/10.1016/j.fmc.2020.11.004

Clark, D., \& Beck, A. (2012). Terapia cognitiva para trastornos de ansiedad. Editorial Desclée de Brouwer, S.A.

Control y la Prevención de Enfermedades. (2021). Afecciones posteriores al COVID-19. https://bit.ly/3HwIWf3

Davis, H., Assaf, G., McCorkell, L., Wei, H., Low, R., Re’em, Y., Redfield, S., Austin, J., \& Akrami, A. (2021). Characterizing long COVID in an international cohort: 7 months of symptoms and their impact. EClinica/Medicine, 38, 34-45. https://doi.org/10.1016/j.eclinm.2021.101019 
Goërtz, Y., Van, M., Delbressine, J., Vaes, A., Meys, R., Machado, F., Houben, S., Burtin, C., Posthuma, R., Franssen, F., Van, N., Hajian, B., Spies, Y., Vijlbrief, H., Van, A., Janssen, D., \& Spruit, M. (2020). Persistent symptoms 3 months after a SARS-CoV-2 infection: the post-COVID-19 syndrome? ERJ Open Research, 6(4), 124-136. https://doi.org/10.1183/23120541.00542-2020

González, F. (2022). Post-COVID-19 conditions in Ecuadorian patients: an observational study. The Lancet Regional Health - Americas, 5, 100088. https://doi.org/10.1016/j.lana.2021.100088

Guerrero, S., \& Bilbao, S. (2021). Persistencia del virus SARS-CoV-2 como causa etiológica de la sintomatología de larga duración en pacientes con COVID-19 persistente. Medicina General y de Familia, 10(2), 85-90. https://doi.org/10.24038/mgyf.2021.027

Higgins, V., Sohaei, D., Diamandis, E., \& Prassas, I. (2021). COVID-19: from an acute to chronic disease? Potential long-term health consequences. Critical Reviews in Clinical Laboratory Sciences, 58(5), 297310. https://doi.org/10.1080/10408363.2020.1860895

López, A., Bernal, M., \& Gómez, R. (2021). Síndrome de COVID-19 persistente. Una revisión narrativa. Revista Clínica Española. https://doi.org/10.1016/j.rce.2021.10.003

Mahmud, R., Rahman, M., Rassel, M., Monayem, F., Sayeed, J., Islam, S., \& Islam, M. (2021). Post-COVID-19 syndrome among symptomatic COVID-19 patients: A prospective cohort study in a tertiary care center of Bangladesh. PLOS ONE, 16(4), e0249644. https://doi.org/10.1371/journal.pone.0249644

National Institute for Health and Care Excellence (NICE). (2020). COVID-19 rapid guideline: managing the longterm effects of COVID-19. https://www.nice.org.uk/guidance/ng188

Organización Mundial de la Salud. (2022). Depresión. https://www.who.int/es/healthtopics/depression\#tab=tab_1

Organizaciones de Naciones Unidas. (2021). Definen el síndrome post COVID-19 o "COVID-19 de larga duración" como enfermedad. Noticias ONU Mirada global Historias humanas. https://bit.ly/36XBf3L

PanAmerican Health Organization. (2021). Impacto de la COVID-19 en los servicios para trastornos mentales, neurológicos y por consumo de sustancias psicoactivas en la región de las Américas: resultados de una evaluación rápida. https://bit.ly/34dBc2Q

Pardo, J., \& López, A. (2021). El síndrome post COVID, incapacidad temporal laboral y prevención. Revista prevención, 2, 123-135. https://bit.ly/3IDimBd

Rodríguez, M., Puchades, F., Ezzeddine, A., Asensio, J., Saiz, C., \& López, M. (2022). Síndrome post cuidados intensivos en COVID-19. Estudio piloto unicéntrico. La calma no llega tras la tempestad. Medicina Clínica, 7, 12-23. https://doi.org/10.1016/j.medcli.2021.11.014

Rodríguez, P., Armenteros, L., Rodríguez, E., \& Gómez, F. (2021). Descripción de los 201 síntomas de la afectación multiorgánica producida en los pacientes afectados por la COVID-19 persistente. Medicina 
General y de Familia, 10(2), 60-68. https://doi.org/10.24038/mgyf.2021.016

Staub, H., \& Staub, L. (2022). Síndrome inflamatória multissistêmica (SIMS) pós-COVID-19. Scientia Medica, 32(1), e42436. https://doi.org/10.15448/1980-6108.2022.1.42436

Valverde, M., González, A., Alvarado, V., \& Miangolarra, J. (2022). Evolución y calidad de vida a los tres meses tras hospitalización por neumonía COVID. Rehabilitación, 14, 1-15. https://doi.org/10.1016/j.r. .2021.11.001

World Health Organization. (2022). COVID-19 weekly epidemiological update (80. ${ }^{a}$ ed.). https://apps.who.int/iris/handle/10665/352199 